\title{
Occurrence and Genetic Correlations of Yersinia spp. Isolated from Commensal Rodents in Northeastern Poland
}

\author{
Aleksandra Platt-Samoraj ${ }^{1, *}$, Klaudia Kończyk-Kmiecik ${ }^{1}$ and Tadeusz Bakuła ${ }^{2} \mathbb{D}$ \\ 1 Department of Epizootiology, Faculty of Veterinary Medicine, University of Warmia and Mazury in Olsztyn, \\ Oczapowskiego 13 Str., 10-718 Olsztyn, Poland; klaudia.konczyk@uwm.edu.pl \\ 2 Department of Veterinary Prevention and Feed Hygiene, Faculty of Veterinary Medicine, \\ University of Warmia and Mazury in Olsztyn, Oczapowskiego 13 Str., 10-718 Olsztyn, Poland; \\ bakta@uwm.edu.pl \\ * Correspondence: platt@uwm.edu.pl
}

Citation: Platt-Samoraj, A.; Kończyk-Kmiecik, K.; Bakuła, T. Occurrence and Genetic Correlations of Yersinia spp. Isolated from Commensal Rodents in Northeastern Poland. Pathogens 2021, 10, 1247. https://doi.org/10.3390/ pathogens 10101247

Academic Editor: Florian Liegeois

Received: 27 August 2021

Accepted: 24 September 2021

Published: 27 September 2021

Publisher's Note: MDPI stays neutral with regard to jurisdictional claims in published maps and institutional affiliations.

Copyright: (c) 2021 by the authors. Licensee MDPI, Basel, Switzerland. This article is an open access article distributed under the terms and conditions of the Creative Commons Attribution (CC BY) license (https:// creativecommons.org/licenses/by/ $4.0 /)$.

\begin{abstract}
Rodents can be a potential Yersinia spp. vector responsible for farm facilities contamination. The aim of the study was to determine the prevalence of Yersinia spp. in commensal rodents found in the farms and fodder factory areas to characterize the obtained isolates and epidemiological risk. Intestinal samples were subjected to bacteriological, bioserotype, and PCR examination for virulence markers ail, yst $A, y s t B$, and inv presence. Yersinia spp. was isolated from 43 out of $244(17.6 \%)$ rodents (Apodemus agrarius $\mathrm{n}=132$, Mus musculus $\mathrm{n}=102$, Apodemus sylvaticus $\mathrm{n}=8$, Rattus norvegicus $\mathrm{n}=2$ ). Y. enterocolitica was isolated from 41 rodents (16.8\%), and from one Y. pseudotuberculosis and one Y. kristensenii. In three cases, two Y. enterocolitica isolates were obtained from one rodent. All $Y$. enetrocolitica contained $y s t B$ and belonged to biotype $1 \mathrm{~A}$, considered as potentially pathogenic. One isolate additionally had the ail gene typical for pathogenic strains. The sequence analysis of the $y s t B$, ail, and inv fragments showed a high similarity to those from clinical cases. The current study revealed a high prevalence of $Y$. enetrocolitica among commensal rodents, but the classification of all of Y. enterocolitica isolates into biotype 1A and the sporadic isolation of Y.pseudotuberculosis do not indicate a high epidemiological risk.
\end{abstract}

Keywords: Yersinia enterocolitica; Yersinia pseudotuberculosis; Yersinia kristensenii; ail; ystB; inv; rodents; vectors

\section{Introduction}

The genus Yersinia belongs to the Enterobacteriaceae family and includes at least 19 species, the list of which is still being revised with newly discovered species [1]. Yersiniosis is a zoonotic gastrointestinal disease caused by two enteropathogenic Yersinia $(Y$.) species, i.e., Y. enterocolitica and Y. pseudotuberculosis [2]. Both pathogens are widely disseminated in the environment. The epidemiology and mechanisms of the circulation of the microorganism are complex and not fully understood. Yersinia spp. can be isolated from animals and food, as well as from water, plants, and soil contaminated by feces of infected animals [3].

According to a recent EFSA (European Food Safety Authority) zoonoses report, yersiniosis was the fourth most frequently reported zoonosis in humans in 2019. There were 6961 confirmed cases in Europe, 648 of which required hospitalization [4]. The disease is usually associated with diarrhea as acute gastroenteritis or pseudoappendicitis, but it can also cause long-term extraintestinal sequels such as erythema nodosum or reactive arthritis. Yersiniosis can also lead to sepsis, which is often fatal [5].

Based on biochemical properties, such as esculin, xylose, and trehalose fermentation, and the production of pyrazinamidase and tween esterase, six biotypes (BT) of Y. enterocolitica were distinguished: $1 \mathrm{~A}, 1 \mathrm{~B}$, and 2, 3, 4, and 5. Depending on the lipopolysaccharide cell wall diversity, over 60 serotypes of $Y$. enterocolitica were distinguished [6-8]. Microorganisms belonging to BT 1B, 2-5 are mainly isolated from clinical cases. Y. enterocolitica 
belonging to BT 1A until recently were considered non-pathogenic because they lack the pathogenicity plasmid pYV (Yersinia virulence plasmid), which contributes to survival and multiplication of bacteria in host tissues, the chromosomal ail and inv genes which encode products responsible for the invasion and adhesion processes to the intestinal epithelial cells, as well as the $y s t A$ encoding enterotoxin YstA [5,9]. However, recent studies have increasingly drawn attention to the potential pathogenic properties of certain strains of the BT 1A which can carry the $y s t B$ gene responsible for the production of YstB enterotoxin [10]. Compared to other Y. enterocolitica biotypes, BT1A is the most heterogeneous and contains the most serotypes $[7,11]$. In the case of $Y$. pseudotuberculosis, all strains isolated from clinical cases are considered pathogenic [12].

For the detection of Yersinia, in addition to traditional bacteriological methods, PCR is commonly used to search for virulence markers, both plasmid, such as myfA, yadA, and chromosomal, such as inv, ail, yst, encoding the production of proteins responsible for the penetration and colonization of the host organism and resistance to the immune mechanisms of the infected organism [9,13].

Yersinia species of clinical importance include the previously mentioned $Y$. enterocolitica and $Y$. pseudotuberculosis as the factors of yersiniosis and $Y$. pestis, which is a plague factor. The remaining Yersinia species, apart from the fish pathogenic Y. ruckeri, are generally considered to be non-pathogenic, conditionally pathogenic or of unknown pathogenetic potential [3]. Many species of livestock, companion animals and free-living animals are susceptible to infection with Yersinia spp. The main reservoir of Y. enterocolitica is considered to be pigs, which often have an asymptomatic carrier and shed the microorganism [14]. Pork meat and food products contaminated with the microorganism excreted by this animal species are considered the most common causes of yersiniosis in humans. The environment in which pigs live is often contaminated with this microorganism, and the elimination of $Y$. enterocolitica from piggeries and their surroundings is hampered by the existence of numerous vectors. One of the most important vectors of the factors are rodents, which can transmit pathogens to domestic animals and to humans [15]. It has been shown that pigs and wild rodents are the reservoirs of the most dangerous high-pathogenic bioserotype 1B/O: 8 Y. enterocolitica in Japan [16].

Compared to $Y$. enterocolitica, much less is known about the transmission pathways and reservoirs of $Y$. pseudotuberculosis, which is less often isolated from livestock and humans but is responsible for the most severe clinical cases of yersiniosis [12].

Commensal rodents are a reservoir for many infectious diseases, but they more often act as a vector. They live in various environments to which they have adapted their body structure and way of life. However, in winter, they inhibit households and livestock facilities. If they are carriers of zoonotic agents, they become a threat to the health of humans, companion animals and farm animals and significantly hinder the control of diseases caused by these pathogens [17].

Recent studies have revealed that rodents have developed unique disease tolerance mechanisms that do not impair their reproductive capacity. It is predicted that they may act as super reservoirs of zoonoses in the future, mainly due to the fact that they can carry several zoonotic pathogens simultaneously. They quickly reach sexual maturity, pregnancies are short, and their offspring are numerous. Additionally, they can spread pathogens over long distances. Rodents that host pathogens may be responsible for transferring them to other buildings within the farm, to neighboring farms and contaminating the environment around them [18]. Feed, which is contaminated at the place of production or storage, is essential in introducing pathogens into herds. In this case, the main blame is also placed on rodents, which, despite biosecurity barriers, often manage to penetrate sites that are rich in food [19].

To date, no studies have been conducted in Poland that could even roughly estimate the importance of commensal rodents which periodically inhabit farm buildings as a vector of pathogenic yersinia and, thus, to what extent they contribute to the occurrence of yersiniosis in humans. The authors' previous research confirmed the circulation of $Y$. enterocolitica 
among wild animals such as beavers and game animals and among small wild forest and field rodents. A high genetic relationship was also demonstrated between yersinia isolated from these animal species [20-22].

The aim of the present research is to determine the prevalence of Yersinia spp.in commensal rodents found in the area of farm facilities and fodder factories and to characterize and analyze the genetic correlations of the obtained isolates to assess the associated public health risk.

\section{Material and Methods}

The study was carried out on 244 rodents obtained from areas belonging to farm buildings and fodder factories in northeastern Poland. The examined facilities included ten locations: four piggeries, two barns and one poultry farm, two fodder factories and an animal house of the Department of Epizootiology, Faculty of Veterinary Medicine, University of Warmia and Mazury in Olsztyn, Poland. The study included 132 striped field mice (Apodemus agrarius), 102 house mice (Mus musculus domesticus), eight field mice (Apodemus sylvaticus), and two brown rats (Rattus norvegicus). In the vicinity of the fodder factories, 107 rodents were collected, 63 originated vicinity of from pig farms, 29 from a vicinity of poultry farm, 43 from vicinity of cattle farms, and 11 rodents were caught in the university animal building.

The animals were caught during deratization campaigns as part of the protection program against pests. Ethical approval was not required because the animals were not sacrificed for research purposes. Rodent control was carried out using Rodenticide bait stations and a snap trap in one facility. The rodents came from two successive fall campaigns from September to December in 2019 and 2020.

Immediately after the animals were delivered to the laboratory, the small intestines were collected, and the rats' livers, kidneys, lungs, line lymph nodes, and spleens were also collected. The samples were crushed and vortexed. Following this, $200 \mu \mathrm{L}$ of each sample suspension was placed in $10 \mathrm{~mL}$ of PSB medium (a peptone sorbitol and bile salts medium prepared according to PN-EN ISO 10273) and incubated at $4{ }^{\circ} \mathrm{C}$ for 21 days. After this time, the culture was transferred by $10 \mu \mathrm{L}$ loop onto CIN agar (Yersinia selective Agar with Yersinia selective agar supplement, MerckKgaA, Germany) in duplicate, with and without alkali treatment. From each tube, $0.5 \mathrm{~mL}$ of culture was placed for $20 \mathrm{~s}$ in $4.5 \mathrm{~mL}$ of $0.5 \%$ $\mathrm{KOH}$ in $0.5 \% \mathrm{NaCl}$, and a loopful of the sample was transferred to the CIN agar and, at the same time, a second loopful was transferred directly from the PBS medium to the agar medium, which was then incubated at $30^{\circ} \mathrm{C}$ for $48 \mathrm{~h}$. For biochemical identification, 1 to 5 typical colonies (a pink to red center surrounded by a transparent border) from each culture were subjected to procedures in accordance with the PN-EN ISO 10273 standard for the initial selection.

BT of Y. enterocolitica was identified according to the method proposed by Wauters [6] and described in the PN-EN ISO 10273 standard [23]. The isolates were tested for the presence of salicin acid production, esculin hydrolysis, xylose acid production, pyrazinamidase activity, and nitrate reduction. The API 20E test (bioMerieux, Marcy-1'Étoile, France) was used to determine the BT of Y. pseudotuberculosis by incubating the test strip at $25^{\circ} \mathrm{C}$ instead of $37^{\circ} \mathrm{C}$ for $20-24 \mathrm{~h}[6,24]$.

Serotypic affiliation was determined on the basis of slide agglutination test results using commercial diagnostic sera O:3, O:5, O:27, O:8, and O:9 (Sifin, Berlin, Germany) according to the manufacturers' guidance. Bacterial cells were obtained from a 24-h blood agar culture (Merc, Berlin, Germany) of examined isolates. Isolates that did not react with any of the sera were designated NI (non-identified).

The course of Multiplex PCR related to the amplification of Y. enterocolitica ail, yst $A$, and $y s t B$ gene fragments was carried out with primers specified in Table 1 . The primers were synthesized in the DNA Sequencing Laboratory of the Polish Academy of Sciences, Oligo, Warsaw, Poland. The reaction was performed using a HotStartTagPlus DNA Polimerase (Qiagen) and a HotStart Master Kit (QIagen). The following PCR protocol was adopted: 
final concentration of $\mathrm{MgCl}_{2}-1.5 \mathrm{nM}$, initial denaturation at $95^{\circ} \mathrm{C}$ for $300 \mathrm{~s}$, followed by 30 cycles of DNA amplification: denaturation at $94^{\circ} \mathrm{C}$ for $45 \mathrm{~s}$, annealing at $54{ }^{\circ} \mathrm{C}$ for $30 \mathrm{~s}$, polymerization at $72{ }^{\circ} \mathrm{C}$ for $60 \mathrm{~s}$, and final polymerization at $72{ }^{\circ} \mathrm{C}$ for $10 \mathrm{~min}$. The reaction was performed in a Mastercycler (Eppendorf, Hamburg, Germany). The products were separated by electrophoresis in 2\% agarose gel with the Midori Green Advanced DNA strain (Nippon Genetics Europe GmbH, Düren, Germany) in 1x TAE buffer. The PCR results were analyzed and archived using the GelDoc gel documentation system (Quantity One analysis software, Bio-Rad, Milan, Italy). The specificity of the reaction was confirmed by sequencing the obtained amplicons.

Table 1. Primer sequences for amplifying ail, yst $A$, yst $B$, and inv genes.

\begin{tabular}{|c|c|c|c|}
\hline Gene & Primer Sequences & Product Size (bp-base pairs) & Source \\
\hline ail & $\begin{array}{l}\text { 5'TGGTTATGCGCAAAGCCATGT3' } \\
\text { 5'TGGAAGTGGGTTGAATTGCA 3' }\end{array}$ & 356 & [25] \\
\hline yst $A$ & $\begin{array}{l}\text { 5'GTCTTCATTTGGAGGATTCGGC3' } \\
\text { 5'AATCACTACTGACTTCGGCTGG3' }\end{array}$ & 134 & [25] \\
\hline$y s t B$ & $\begin{array}{l}\text { 5'TGTCAGCATTTATTCTCAACT3' } \\
\text { 5'GCCGATAATGTATCATCAAG3' }\end{array}$ & 180 & [26] \\
\hline$i n v$ & $\begin{array}{l}\text { CGGTACGGCTCAAGTTAATCTG } \\
\text { CCGTTCTCCAATGTACGTATCC }\end{array}$ & 183 & [9] \\
\hline
\end{tabular}

Nucleotides were sequenced with longer ystB primers (263bp) [YSTBF- $5^{\prime}$ GGA CAC CGC ACA GCT TAT ATT TT 3', YSTBR - 5' GCA CAG GCA GGA TTG CAA CA 3'], while for inv sequencing, new primers were developed, using the Prime Blast program, for longer amplicons (567 bp): INVF 1: -5 'GGCAGATCCTATTCCAGATG-3', INVR 2: $5^{\prime}$ CTCACCGAATAACTTGGGAA- ${ }^{\prime}$ ). The amplicons were cleaned with the Klin-up Purification Kit (A\&A Biotechnology, Gdańsk, Poland) according to the manufacturer's recommendations. Purified amplicons were directly sequenced in both directions (Genomed S.A., Poland). Multiple sequence alignment was carried out in CLUSTAL W [27]. Nucleotide and amino acid sequences were identified using BIOEDIT v.7.2.0 software. The nucleotide sequences of $y s t B$, ail and inv analyzed in this study are available in the GeneBank [MZ496229MZ496272] and [MZ491080-MZ491082].

\section{Results}

Yersinia spp. was isolated from intestinal samples from $17.6 \%(n=43 / 244)$ of the tested animals, including Y. enterocolitica from $16.8 \%$ of the rodents $(n=41 / 244)$ and $Y$. pseudotuberculosis from one M. musculus and $Y$. kristensenii also from one M. musculus. In three cases, two different $Y$. enterocolitica isolates were obtained from two A. agrarius and one $R$. norvegicust. In total, 46 isolates were subjected to further studies. The results of the bacteriological study for the presence of Yersinia spp. confirmed by PCR are presented in Table 2.

Table 2. Characteristics of the Yersinia spp. isolates.

\begin{tabular}{ccccccc}
\hline Isolate No. & Acc. No. & Yersinia & Gene & Biotype & Serotype & Source/Isolate Name \\
\hline 1. & MZ496229 & Y.enterocolitica & ystB & $1 \mathrm{~A}$ & O:5 & 9 (Mus musculus) \\
\hline 2. & MZ496230 & Y.enterocolitica & ystB & $1 \mathrm{~A}$ & O:5 & 11 (Apodemus agrarius) \\
\hline 3. & MZ496231 & Y.enterocolitica & ystB & $1 \mathrm{~A}$ & NT & 12 (Mus musculus) \\
\hline 4. & MZ496232 & Y.enterocolitica & ystB & $1 \mathrm{~A}$ & NT & 41 (Mus musculus) \\
\hline 5. & MZ496233 & Y.enterocolitica & ystB & $1 \mathrm{~A}$ & $\mathrm{NT}$ & 53 (Mus musculus) \\
\hline 6. & MZ491080 & Y. kristensenii & ail & & - & 54 (Mus musculus) \\
\hline
\end{tabular}


Table 2. Cont.

\begin{tabular}{|c|c|c|c|c|c|c|}
\hline Isolate No. & Acc. No. & Yersinia & Gene & Biotype & Serotype & Source/Isolate Name \\
\hline 7. & MZ496234 & Y. enterocolitica & $y s t B$ & $1 \mathrm{~A}$ & NT & 56 (Mus musculus) \\
\hline 8. & MZ491082 & Y.pseudotuberculosis & $i n v$ & I & - & 70 (Mus musculus) \\
\hline 9. & MZ496235 & Y. enterocolitica & $y s t B$ & $1 \mathrm{~A}$ & NT & 100 (Apodemus agrarius) \\
\hline 10. & MZ496236 & Y. enterocolitica & ystB & $1 \mathrm{~A}$ & NT & 102 (Apodemus agrarius) \\
\hline 11. & MZ496237 & Y. enterocolitica & $y s t B$ & $1 \mathrm{~A}$ & NT & 107 (Mus musculus) \\
\hline 12. & MZ496238 & Y. enterocolitica & $y s t B$ & $1 \mathrm{~A}$ & NT & 108 (Mus musculus) \\
\hline 13. & MZ496239 & Y. enterocolitica & $y s t B$ & $1 \mathrm{~A}$ & NT & 110 (Apodemus agrarius) \\
\hline 14. & MZ496240 & Y. enterocolitica & $y s t B$ & $1 \mathrm{~A}$ & NT & 114 (Mus musculus) \\
\hline 15. & MZ496241 & Y. enterocolitica & $y s t B$ & $1 \mathrm{~A}$ & NT & 142 (Mus musculus) \\
\hline 16. & MZ496242 & Y. enterocolitica & $y s t B$ & $1 \mathrm{~A}$ & NT & 143 (Apodemus agrarius) \\
\hline 17. & MZ496243 & Y. enterocolitica & ystB & $1 \mathrm{~A}$ & NT & 144 (Apodemus agrarius) \\
\hline 18. & MZ496244 & Y. enterocolitica & $y s t B$ & $1 \mathrm{~A}$ & NT & 146 (Mus musculus) \\
\hline 19. & MZ496245 & Y. enterocolitica & $y s t B$ & $1 \mathrm{~A}$ & NT & 147 (Apodemus agrarius) \\
\hline 20. & MZ496246 & Y. enterocolitica & ystB & $1 \mathrm{~A}$ & NT & 148 (Mus musculus) \\
\hline 21. & MZ496247 & Y. enterocolitica & $y s t B$ & $1 \mathrm{~A}$ & NT & 149 (Apodemus agrarius) \\
\hline 22. & MZ496248 & Y. enterocolitica & ystB & $1 \mathrm{~A}$ & NT & 150 (Mus musculus) \\
\hline 23. & MZ496249 & Y. enterocolitica & ystB & $1 \mathrm{~A}$ & NT & 151 (Mus musculus) \\
\hline 24. & MZ496250 & Y. enterocolitica & $y s t B$ & $1 \mathrm{~A}$ & NT & 152 (Apodemus agrarius) \\
\hline 25. & MZ496251 & Y. enterocolitica & ystB & $1 \mathrm{~A}$ & NT & 154 (Mus musculus) \\
\hline 26. & MZ496252 & Y. enterocolitica & $y s t B$ & $1 \mathrm{~A}$ & O:5 & 164 (Apodemus agrarius) \\
\hline 27. & MZ496253 & Y. enterocolitica & $y s t B$ & $1 \mathrm{~A}$ & NT & 170 (Mus musculus) \\
\hline 28. & MZ496254 & Y. enterocolitica & $y s t B$ & $1 \mathrm{~A}$ & NT & 178 (Mus musculus) \\
\hline 29. & MZ496255 & Y. enterocolitica & ystB & $1 \mathrm{~A}$ & NT & 182 (Mus musculus) \\
\hline 30. & MZ496256 & Y. enterocolitica & $y s t B$ & $1 \mathrm{~A}$ & O:5 & 183 (Mus musculus) \\
\hline 31. & MZ496257 & Y. enterocolitica & $y s t B$ & $1 \mathrm{~A}$ & NT & 201 (Apodemus agrarius) \\
\hline 32. & MZ496258 & Y. enterocolitica & ystB & $1 \mathrm{~A}$ & NT & 202 (Apodemus agrarius) \\
\hline 33. & MZ496259 & Y. enterocolitica & ystB & $1 \mathrm{~A}$ & NT & 203a (Apodemus agrarius) \\
\hline 34. & $\begin{array}{l}\text { MZ496260 } \\
\text { MZ491081 }\end{array}$ & Y. enterocolitica & ystB, ail & $1 \mathrm{~A}$ & $\mathrm{O}: 3$ & 203b (Apodemus agrarius) \\
\hline 35. & MZ496261 & Y. enterocolitica & ystB & $1 \mathrm{~A}$ & NT & 204a (Rattus norvegicus) \\
\hline 36. & MZ496262 & Y. enterocolitica & $y s t B$ & $1 \mathrm{~A}$ & NT & 204b (Rattus norvegicus) \\
\hline 37. & MZ496263 & Y. enterocolitica & $y s t B$ & $1 \mathrm{~A}$ & NT & 205 (Apodemus agrarius) \\
\hline 38. & MZ496264 & Y. enterocolitica & $y s t B$ & $1 \mathrm{~A}$ & NT & 206 (Apodemus agrarius) \\
\hline 39. & MZ496265 & Y. enterocolitica & $y s t B$ & $1 \mathrm{~A}$ & NT & 207 (Apodemus agrarius) \\
\hline 40. & MZ496266 & Y. enterocolitica & ystB & $1 \mathrm{~A}$ & O:5 & 209a (Apodemus agrarius) \\
\hline 41. & MZ496267 & Y. enterocolitica & $y s t B$ & $1 \mathrm{~A}$ & NT & 209b (Apodemus agrarius) \\
\hline 42. & MZ496268 & Y. enterocolitica & ystB & $1 \mathrm{~A}$ & NT & 213 (Apodemus agrarius) \\
\hline 43. & MZ496269 & Y. enterocolitica & ystB & $1 \mathrm{~A}$ & NT & 223 (Apodemus agrarius) \\
\hline 44. & MZ496270 & Y. enterocolitica & ystB & $1 \mathrm{~A}$ & $\mathrm{O}: 8$ & 227 (Apodemus agrarius) \\
\hline 45. & MZ496271 & Y. enterocolitica & $y s t B$ & $1 \mathrm{~A}$ & $\mathrm{O}: 8$ & 228 (Apodemus agrarius) \\
\hline 46. & MZ496272 & Y. enterocolitica & $y s t B$ & $1 \mathrm{~A}$ & NT & 244 (Rattus norvegicus) \\
\hline
\end{tabular}


Of the 102 examined M. musculus, 18 were Y. enterocolitica-positive (17.6\%, $\mathrm{n}=18 / 102)$. A slightly lower percentage of infections was found among $A$. agrarius, in which, out of 132 examined mice, Y. enterocolitica was found in 21 mice $(15.9 \%, n=132 / 21)$. Y. enterocolitica was isolated from intestinal samples of both examined rats. The bacteria were also cultured from rat internal organs, i.e., the liver, lungs, kidneys, mesenteric lymph nodes, and spleen from one rat and the liver and mesenteric lymph nodes from another individual. Yersinia spp. were not isolated from A. sylvaticus.

PCR showed the presence of $y s t B$ in all $Y$. enterocolitica isolates. One isolate from $A$. agrarius had the two virulence markers, ail and $y s t B$. The only isolate identified by API $20 \mathrm{E}$ as $Y$. kristensenii contained only the ail gene. The $y s t A$ gene was not found in any of the isolates.

Yersinia isolates confirmed by PCR were subjected to sero/biotyping. All obtained Y. enterocolitica isolates were salicyl-, eskulin-, and xylose-positive and since they exhibited the presence of pyrazinamidase activity and nitrate reduction, they were classified as BT 1A (Ye behavior). The inv-positive Y. pseudotuberculosis was classified as BT I (raffinose fermentation, melibiose fermentation and metabolic conversion of citrate) according to Niskanen et al. [24] and Tsubokura and Aleksic [4].

The results of the serotype affiliation are presented in Table 2. The majority of the 36 isolates did not react with any of the available diagnostic sera and were defined as NI (non-identified).

The serotyping results confirmed the differentiation of double isolates from A. agrarius 203a and 203bApodemus agrarius (NI and O:3, respectively) and 209a and 209bApodemus agrarius (O:5 and NI, respectively). For isolates, 204a and bRattus norvegicus serotypes were not established (Table 2).

Sequencing results confirmed the species affiliation of the isolates. Most of the sequenced $y s t B$ fragments were contained in two clusters. Only the $y s t B$ sequences of the 56Mus musculus and 205Apodemus agrarius isolates were located in separate branches (Figure 1).

The evolutionary history was inferred using the UPGMA method [28]. The optimal tree is shown. The tree is drawn to scale, with branch lengths in the same units as those of the evolutionary distances used to infer the phylogenetic tree. The evolutionary distances were computed using the Maximum Composite Likelihood method [29] and are in the units of the number of base substitutions per site. This analysis involved 44 nucleotide sequences. Codon positions included were $1 \mathrm{st}+2 \mathrm{nd}+3 \mathrm{rd}+$ Noncoding. All ambiguous positions were removed for each sequence pair (pairwise deletion option). There were a total of 264 positions in the final dataset. Evolutionary analyses were conducted in MEGA X [30].

The results of the sequencing of $y s t B$ fragments confirmed the differentiation of the double-isolated isolates from the field mice 209aApodemus agrarius and 209bApodemus agrarius, and 204aRattus Norvegicus and 204b from the rat. In both cases, sequences "a" and " $b$ " were located in different clusters. The sequences of $y s t B$ fragments in both isolates from the field mouse 203aApodemus agrarius and 203b were the same, the difference was that the isolate 203aApodemus agrarius also contained the ail gene.

The partial coding sequence (cds) of ystB 263 base pairs (bp) long, from $11 \mathrm{M}$. musculus [MZ496229, MZ496231, MZ496241, MZ496244, MZ496246, MZ496248, MZ496251, MZ496253-MZ496256], 15 striped field mice [MZ496230, MZ496236, MZ496242, MZ496243, MZ496245, MZ496247, MZ496250, MZ496252, MZ496257-MZ496260, MZ496266, MZ496270, MZ496271] and from 1 R. norvegicus [MZ496261] were grouped in one cluster and all of these sequences demonstrated $100 \%$ similarity to the $y s t B$ sequences in $Y$. enterocolitica isolated from a beaver [Acc. No. KJ592623] in northern Poland, common voles [Acc. No. MK734430, MK734429.1] in southeastern Poland, from a food sample in South Korea [Acc No CP009456.1], and from a human host fecal sample in the UK [Acc. No. HF571988.1]. 


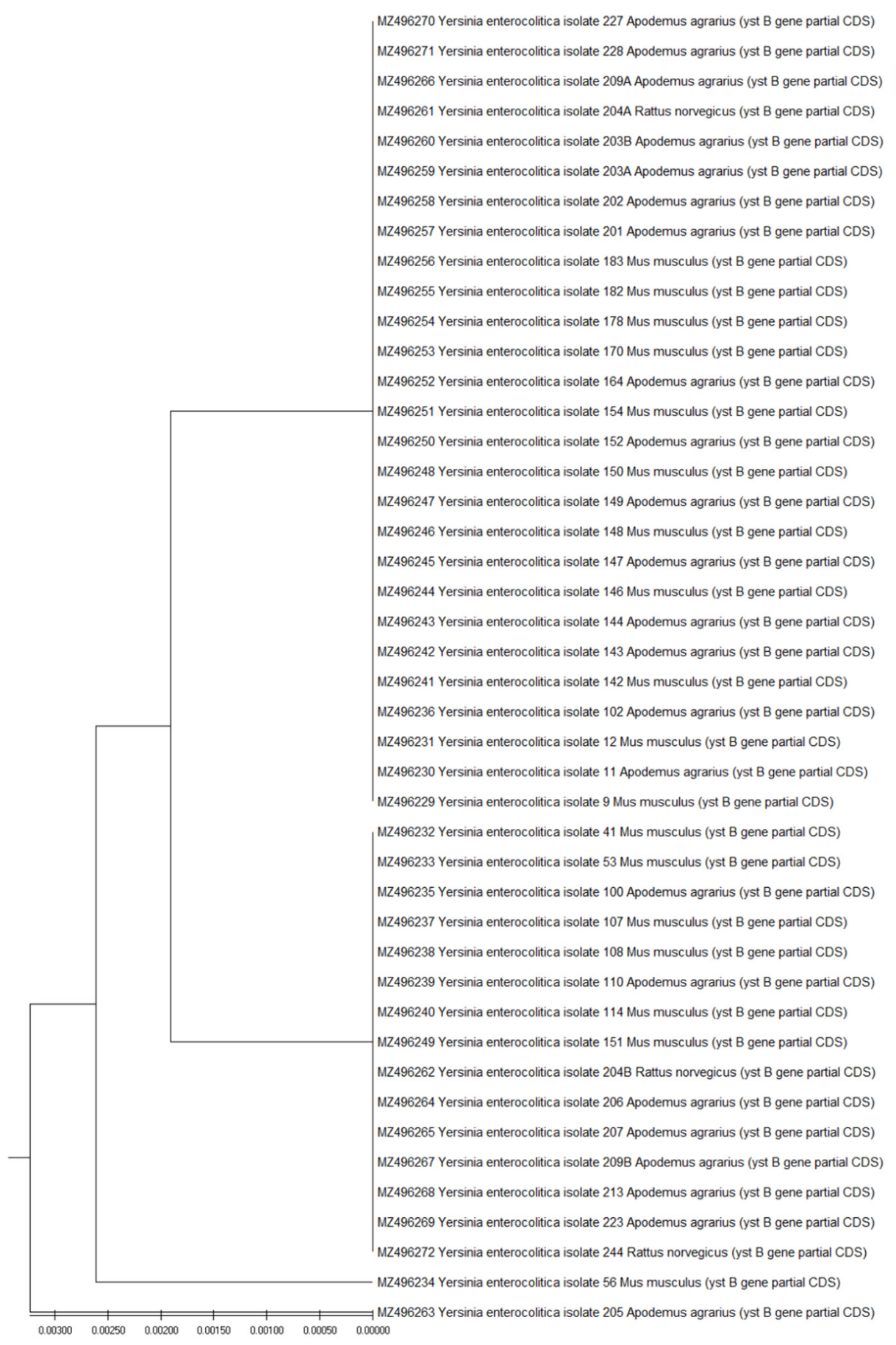

Figure 1. Evolutionary relationships of taxa.

The second group of sequences was located in a separate cluster. The cds of $y s t B$ from six M. musculus [MZ496232, MZ496233, MZ496237, MZ496238, MZ496240, MZ496249], from seven A. agrarius [MZ496235, MZ496239, MZ496264, MZ496265, MZ496267-MZ496269], and from two R. norvegicus [MZ496262, MZ496272] demonstrated 100\% similarity to the ystB sequences in $Y$. enterocolitica isolated from common voles [Acc. No. MK734428.1, MK734423.1, MK734422.1], yellow naked mice [MK734426.1, MK734425.1, MK734424.1, MK734422.1] in southwestern Poland and from a beaver in northern Poland [KJ592624.1].

The cds of the first of the separately located $y s t B$ fragments from the M. musculus isolate [MZ496234] had no Gene Bank counterpart with 100\% similarity, but they demonstrated $99.625 \%$ similarity to the $y s t B$ sequences in $Y$. enterocolitica isolated from common voles [Acc. No. MK734430.1, MK734429.1] in southeastern Poland and a human host fecal sample in 
UK [HF571988.1] and 98.48\% similarity to $y s t B$ from a mallard duck in northern Poland [KU198401.1]. The second different sequence from A. agrarius [MZ496263] showed 100\% similarity to the $y s t B$ sequence in enterotoxin producing $Y$. enterocolitica $1 \mathrm{~A}$ strain in India [Acc. No. AY966880.1], beaver [Acc. No. KJ592627.1] in Poland, and 99.62\% similarity to sequences from common voles [Acc. No. MK734428.1, MK734423.1] and yellow naked mice [Acc. No. MK734424.1, MK734425.1, MK734427.1] from southeastern Poland.

Partial cds analysis of the ail gene ( $356 \mathrm{bp}$ ) from $Y$. enterocolitica isolated from A. agrarius showed $100 \%$ similarity to the sequences derived from Y. enterocolitica $1 \mathrm{~A}$ isolated from a raw pork sample in Germany [Acc. No. FR847859.1], a clinical stool sample in Finland [Acc. No. FN812732.1], and a wild boar in Poland [Acc. No. KM253257.1].

A partial cds analysis of the ail gene from Y. kristensenii isolated from M. musculus showed $76.55 \%$ similarity to the sequence derived from $Y$. enetrocolitica isolated from rats in China [Acc. No. JX972144.1] and 74.72\% similarity to the ail sequence from Y. enterocolitica isolated from raw pork in Germany [Acc. No. FR847859.1], Y. enterocolitica isolated from clinical stool sample in Finland [Acc. No. FN812732.1] and 74.44\% similarity to a sequence from Y. enterocolitica isolated from fatal septicemia in the USA [Acc. No. CP009846.1].

The partial cds of inv (567 bp), [Acc. No. MZ491082] from Y. pseudotuberculosis isolate was 100\% identical to corresponding sequences of Y. pseudotuberculosis BTI strain isolated from a clinical human sample in France [Acc. No. CP033713.1], to invasive strains from Finland [Acc. No.HE805230.1-HE805218.1] and to a corresponding sequence of $Y$. pseudotuberculosis isolated from a striped field mouse in southeastern Poland (Acc. No. MZ491083).

\section{Discussion}

This is the first study on the prevalence and genetic analysis of isolates of Yersinia spp. isolated from commensal rodents in Poland. The authors' previous research concerned the occurrence of these pathogens among species of small wild rodents inhabiting forest and field environments, which usually hibernate in winter and rarely reach households or farm buildings.

The presented study showed widespread Y. enterocolitica among rodents potentially inhabiting farm objects. The presented research did not cover the situation inside these facilities. The presence of Yersinia spp in $17.6 \%$ of samples of rodents included in the study proves their important role in the transmission of bacteria. Although the isolates of Y. enterocolitica obtained in the current study contained the $y s t B$ gene (characteristic of strains belonging to BT 1A and commonly considered non-pathogenic), based on the literature data rodents can also be infected with pathogenic strains. In a study by Backhans et al. [31] in which 190 colon samples were tested by PCR, Y. enterocolitica was isolated from $5 \%$ of mice caught in the vicinity of pig farms. The obtained isolates included, among others, those belonging to the 4/O:3 bioserotypes. Hayashidani et al. [17] and Oda et al. [32] isolated highly virulent O:8, BT 1B bioserotypes of $Y$. enterocolitica from field mice with the same pulsotypes as those isolated from pigs.

In comparing the authors' own results with results reported in other studies on yersinia prevalence in small rodents, similar results were obtained in Great Britain, Scandinavian countries, Germany and France, where mainly isolates belonging to BT 1A were found in domestic mice [19,33-35]. Oda et al. [32] obtained a percentage of positive Y. enterocolitica samples, similar to the current study, where out of 560 tested animals, $15.7 \%$ of wild rodents showed the presence of this microorganism.

The current study showed that rodents may be carriers of more than one strain of Y. enterocolitica. In three cases, two isolates were obtained from one animal serotypically and genotypically different from each other. Particularly noteworthy is the case of isolating two Y. enetreocolitica BT 1A isolates from one A. agrarius. The first isolate contained the $y s t B$ virulence marker typical for this biotype (203a Apodemus agrarius), while the second isolate, (203b Apodemus agrarius), contained $y s t B$ with the same nucleotide sequences as the first isolate but additionally had the ail virulence marker. This is interesting because the ail gene, controlling the Ail protein, which plays an important role during attachment 
and invasion processes, usually occurs in tandem with the $y s t A$ gene and is typical for pathogenic Y. enterocolitica biotypes 2-5 [36]. The comparison of the ail sequence of the isolate in the current study with that available in the GeneBank database showed $100 \%$ similarity to the sequence detected in Y. enterocolitica isolated from a clinical stool sample from Finland [Acc. No. FN812732.1]. This may suggest potential pathogenic properties of ail/ystB-positive strains. Therefore, the question arises of what mechanisms of attaching additional genetic structures are responsible for the formation of such previously unknown Y. enterocolitica strains and, above all, how it affects the pathogenetic abilities of the bacterium. Undoubtedly, this is a problem that deserves more in-depth research. To the best of our knowledge this is the first case of the detection of such an atypical isolate from a rodent, which is simultaneously a carrier of the typical isolate $Y$. enterocolitica BT 1A. The authors previously discussed the occurrence of such rare ail/ystB-positive isolates detected in game animals in Poland [37].

The isolate found in one M. musculus, identified by API20E with $89.2 \%$ probability as Y. kristensenii, also contained the ail gene, and the sequences of its fragment differed from the ail sequences available in GeneBank database. In the available literature, there was only one article describing the ail presence in Y. kristensenii [38]. However, the isolate mentioned above did not show the simultaneous presence of the yst gene, unlike those reported by Joutsen et al. [38]. According to the authors cited above, the identification of $Y$. kristensenii based on the API20E result is subject to a certain margin of error. The result of sequencing the ail fragment of the isolate in the current study confirmed that it belongs to the genus Yersinia, but the exact determination of the species requires more detailed analyses.

In the current study, the results of the sequencing of $y s t B$ fragments showed relatively little variability. Importantly, the comparison of the studied sequences with the data available in the NCBI database showed no similarities to the analogous ystB fragments derived from $Y$. enterocolitica isolated from farm animals. This may be due to both the lack of correlation and the lack of availability of similar sequences in the NCBI database. Additionally, this result supports extending the research in the future by comparing isolates from rodents with isolates obtained from farm animals from the areas where these studies were conducted.

Y. pseudotuberculosis, the second yersinia co-responsible for yersiniosis, due to its history, may be associated with rodents, as it was previously thought to be the cause of the disease known as rodenciosis [39]. Fukushima et al. identified pest rodents as an animal factor associated with a high risk of $Y$. pseudotuberculosis on pig farms [40]. The results of the current study confirm that rodents can also be carriers of $Y$. pseudotuberculosis, which is responsible for the most severe cases of yersiniosis, but there are no records in the literature confirming that this yersinia was more often isolated from rodents than from other animal species. Contrary to expectations, in the current study it was successfully isolated from only one house mouse. The results of the experiments of other authors indicate difficulties in the culturing of this bacteria [33]. Thus, such a small number of isolations may be partially due to the limitations of laboratory methods. These assumptions are confirmed by the results of the authors' previous studies and studies by other authors. In a previous study by the authors, only one isolate of $Y$. pseudotuberculosis was isolated in 214 wild forest rodents [22]. In a study by Backhans et al. [33], similar to the current results, out of 190 colon samples from rodents, Y. pseudotuberculosis was detected in only one animal.

It is more difficult to lure rats to rodenticide bait stations routinely used in rodent control actions. Rat invasions periodically occur in livestock farms but not as frequently as mice invasions, which are seasonal in nature. Therefore, only two R. norvegicus were provided for the current study and they were caught in the areas of two different feed factories. It is noteworthy that the two rats that were lured into the traps were both infected with Y. enterocolitica and the bacteria was isolated from many of their internal organs.

It is believed that because of the rodents that transmit the yersinia that it is impossible to obtain Yersinia-free herds in pig farms, despite following the strictest biosecurity rules $[14,33]$. The high prevalence percentage of yersinia in rodents in the current study 
may be related to the seasonality of rodent infestation in farm buildings occurring at the turn of autumn and winter. This coincides with a period of more frequent isolation of Y. enterocolitica, which has psychrophilic properties. However, none of the isolates obtained in the current study belonged to the so-called classic pathogenic biotypes that pose a threat to human health.

\section{Conclusions}

The current study revealed a high prevalence of $Y$. enetrocolitica among commensal rodents. Although field mice were caught more frequently in the vicinity of farm buildings than domestic mice, Y. enterocolitica was more often isolated from intestinal samples of domestic mice. The classification of all obtained isolates into BT 1A of Y. enterocolitica and the sporadic isolation of $Y$. pseudotuberculosis do not indicate a high share of commensal rodents in the spreading of yersiniosis. However, the current study revealed that rodents may be carriers of more than one strain of Y. enterocolitica, including a new kind of BT 1A isolate with as yet unexplained pathogenic properties with the ail virulence marker.

Author Contributions: Conceptualization, A.P.-S.; funding acquisition, A.P.-S. and T.B.; field investigation, T.B.; laboratory methodology, A.P.-S. and K.K.-K.; writing-original draft preparation, A.P.-S., K.K.-K.; writing-review and editing, A.P.-S., K.K.-K. and T.B. All authors have read and agreed to the published version of the manuscript.

Funding: Project financially co-supported by Minister of Science and Higher Education in the range of the program entitled "Regional Initiative of Excellence" for the years 2019-2022, Project No. 010/RID/2018/19.

Institutional Review Board Statement: The animals were caught during deratization campaigns as part of the protection program against pests. Ethical approval was not required because the animals were not sacrificed for research purposes.

Informed Consent Statement: Not applicable.

Data Availability Statement: Not applicable.

Acknowledgments: The Authors thank Anna Szczerba-Turek for the implementation and description of Figure 1. and for the registration of sequences in the GeneBank.

Conflicts of Interest: The authors declare no conflict of interest.

\section{References}

1. Nguyen, S.V.; Muthappa, D.M.; Hurley, D.; Donoghue, O.; McCabe, E.; Anes, K.; Schaffer, J.; Murphy, B.P.; Buckley, J.F.; Fanning, S. Yersinia hibernica sp. nov., isolated from pig-production environments. Int. J. Syst. Evol. Microbiol. 2019, 7, 2023-2027. [CrossRef]

2. EFSA. Monitoring and identification of human enteropahogenic Yersinia spp. EFSA J. 2007, 595, 1-30.

3. Sulakvelidze, A. Yersiniae other than Y. enterocolitica, Y. pseudotuberculosis, and Y. pestis: The ignored species. Microbes Infect. 2000, 2, 497-513. [CrossRef]

4. The European Union One Health 2019 Zoonoses Report. EFSA J. 2021, 19, 6404. [CrossRef]

5. Bottone, E.J. Yersinia enterocolitica: The charisma continues. Clin. Microbiol. Rev. 1997, 10, 257-276. [CrossRef]

6. Tsubocura, M.; Aleksic, S. A simplified antigenic scheme for serotyping of Yersinia pseudotuberculosis: Phenotypic characterization of reference strains and preparation of $\mathrm{O}$ and $\mathrm{H}$ factor sera. Contrib. Microbiol. Immunol. 1995, 13, 99-105.

7. Bari, L.; Hossain, M.A.; Isshiki, K.; Ukuku, D. Behavior of Yersinia enterocolitica in Foods. J. Pathog. 2011, 2011, 420732. [CrossRef] [PubMed]

8. Wauters, G.; Kandolo, K.; Janssens, M. Revised biogrouping scheme of Yersinia enterocolitica. Contrib. Microbiol. Immunol. 1987, 9, 14-21. [PubMed]

9. Thoerner, P.; Bin Kingombe, C.I.; Bogli-Stuber, K.; Bissing-Choisat, B.; Wassenaar, T.M.; Frey, J.; Jemmi, T. PCR detection of virulence genes in Yersinia enterocolitica and Yersinia pseudotuberculosis and investigation of virulence gene distribution. Appl. Environ. Microbiol. 2003, 69, 1810-1816. [CrossRef]

10. Campioni, F.; Falcão, J.P. Genotypic diversity and virulence markers of Yersinia enterocolitica biotype 1A strains isolated from clinical and non-clinical origins. APMIS 2014, 122, 215-222. [CrossRef]

11. Wren, B. The Yersiniae-A model genus to study the rapid evolution of bacterial pathogen. Nat. Rev. Microbiol. 2003, 1, 55-64. [CrossRef] 
12. Amphlett, A. Far East Scarlet-Like Fever: A Review of the Epidemiology, Symptomatology, and Role of Superantigenic Toxin: Yersinia pseudotuberculosis-Derived Mitogen A. Open Forum Infect. Dis. 2015, 3, ofv202. [CrossRef]

13. Savin, C.; Criscuolo, A.; Guglielmini, J.; Le Guern, A.-S.; Carniel, E.; Pizarro-Cerdá, J.; Brisse, S. Genus-wide Yersinia core-genome multilocus sequence typing for species identification and strain characterization. Microb. Genom. 2019, 5, e000301. [CrossRef]

14. Gurtler, M.; Alter, T.; Kasimir, S.; Fehlhaber, K. Prevalence of Yersinia enterocolitica in fattening pigs. J. Food. Prot. 2005, 68, 850-854. [CrossRef]

15. Backhans, A.; Jacobson, M.; Hansson, I.; Lebbad, M.; Thisted Lambertz, S.; Gammelgård, E.; Saager, M.; Akande, O.; Fellström, C. Occurrence of pathogens in wild rodents caught on Swedish pig and chicken farms. Epidemiol. Infect. 2013, 141, $1885-1891$. [CrossRef] [PubMed]

16. Hayashidani, H.; Ishiyama, Y.; Okatani, T.A.; Yoshida, S.; Ishikawa, M.; Kato, Y.; Ohtomo, Y.; Saito, M.; Horisaka, T.; Kaneko, K.; et al. Molecular genetic typing of Yersinia enterocolitica serovar O:8 isolated in Japan. Adv. Exp. Med. Biol. 2003, 529, 363-365. [PubMed]

17. Kruse, H.; Kirkemo, A.M.; Handeland, K. Wildlife as a source of zoonotic infections. Emerg. Infect. Dis. 2004, 10, $2067-2072$. [CrossRef]

18. Han, B.A.; Schmidt, J.P.; Bowden, S.E.; Drake, J.M. Rodent reservoirs of future zoonotic diseases. Proc. Natl. Acad. Sci. USA 2015, 112, 7039-7044. [CrossRef]

19. Pockock, M.J.O.; Searle, J.B.; Betts, W.B.; White, P.C.L. Patterns of infection by Salmonella and Yersinia spp. in commensal house mouse (Mus musculus domesticus) populations. J. Appl. Microbiol. 2001, 90, 755-760. [CrossRef] [PubMed]

20. Syczyło, K.; Platt-Samoraj, A.; Bancerz-Kisiel, A.; Szczerba-Turek, A.; Pajdak, J.; Łabuć, S.; Procajło, Z.; Socha, P.; Chuzhebayeva, G.; Szweda, W. The prevalence of Yersinia enterocolitica in game animals in Poland. PLoS ONE 2018, 13, e0195136. [CrossRef] [PubMed]

21. Platt-Samoraj, A.; Syczyło, K.; Bancerz-Kisiel, A.; Szczerba-Turek, A.; Giżejewska, A.; Szweda, W. Yersinia enterocolitica strains isolated from beavers (Castor fiber). Pol. J. Vet. Sci. 2015, 18, 449-451. [CrossRef]

22. Platt-Samoraj, A.; Żmudzki, J.; Pajdak-Czaus, J.; Szczerba-Turek, A.; Bancerz-Kisiel, A.; Procajło, Z.; Łabuć, S.; Szweda, W. The Prevalence of Yersinia enterocolitica and Yersinia pseudotuberculosis in small wild rodents in Poland. Vector-Borne Zoonotic Dis. 2020, 20, 586-592. [CrossRef] [PubMed]

23. PN-EN ISO 10273: Microbiology of Food and Animal Feed. Horizontal Method for the Detection of Microbiology of Food and Animal Feed. Horizontal Method for the Detection of Presumably Pathogenic Yersinia enterocolitica. 2005. Polish Committee for Standardization: Polish Norm-European Norm (with appendix PN-EN ISO 10273:2005/Ap1, 2005; PN-EN ISO 10273:2005/Ap2, 2006). Available online: https: / / www.iso.org/standard/34564.html (accessed on 27 August 2021).

24. Niskanen, T.; Laukkanen, R.; Murros, A.; Björkroth, J.; Skurnik, M.; Korkeala, H.; Fredriksson-Ahomaa, M. Characterisation of non-pathogenic Yersinia pseudotuberculosis-like isolated from food and environmental samples. Int. J. Food Microbiol. 2009, 129, 150-156. [CrossRef] [PubMed]

25. Harnett, N.; Lin, Y.P.; Krishan, C. Detection of pathogenic Yersinia enterocolitica using the multiplex polymerase chain reaction. Epidemiol. Infect. 1996, 117, 59-69. [CrossRef]

26. Platt-Samoraj, A.; Ugorski, M.; Szweda, W.; Szczerba-Turek, A.; Wojciech, Ł.; Procajło, Z. Analysis of the Presence of ail, ystA and $y s t B$ genes in Yersinia enterocolitica strains isolated from aborting sows and aborted fetuses. J. Vet. Med. B 2006, 53, 341-346. [CrossRef] [PubMed]

27. Larkin, M.A.; Blackshields, G.; Brown, N.P.; Chenna, R.; McGettigan, P.A.; McWilliam, H.; Valentin, F.; Wallace, I.M.; Wilm, A.; Lopez, R.; et al. Clustal W and Clustal X version 2.0. Bioinformatics 2007, 23, 2947-2948. [CrossRef] [PubMed]

28. Sneath, P.H.A.; Sokal, R.R. Numerical Taxonomy; Freeman: San Francisco, CA, USA, 1973.

29. Tan, S.Y.; Dutta, A.; Jakubovics, N.S.; Ang, M.Y.; Siow, C.C.; Mutha, N.V.R.; Heydari, H.; Wee, W.Y.; Wong, G.J.; Choo, S.W. YersiniaBase: A genomic resource and analysis platform for comparative analysis of Yersinia. BMC Bioinform. 2015, 16, 9. [CrossRef]

30. Kumar, S.; Stecher, G.; Li, M.; Knyaz, C.K. MEGA X: Molecular Evolutionary Genetics Analysis across computing platforms. Mol. Biol. Evol. 2018, 35, 1547-1549. [CrossRef]

31. Backhans, A.; Fellström, C. Rodents on pig and chicken farms-A potential threat to human and animal health. Infect. Ecol. Epidemiol. 2012, 2, 1793. [CrossRef]

32. Oda, S.; Kabeya, H.; Sato, S.; Shimonagane, A.; Inoue, K.; Hayashidani, H.; Takada, N.; Fujita, H.; Kawabata, H.; Maruyama, S. Isolation of pathogenic Yersinia enterocolitica 1B/O:8 from Apodemus mice in Japan. J. Wildl. Dis. 2015, 51, 260-264. [CrossRef]

33. Backhans, A.; Fellström, C.; Lambertz, S.T. Occurrence of pathogenic Yersinia enterocolitica and Yersinia pseudotuberculosis in small wild rodents. Epidemiol. Infect. 2011, 139, 1230-1238. [CrossRef] [PubMed]

34. Kapperud, G. Yersinia enterocolitica in small rodents from Norway, Sweden and Finland. Acta Pathol. Microbiol. Immunol. Scand. B 1975, 83B, 335-342. [CrossRef] [PubMed]

35. Servan, J.; Brault, J.; Alonso, J.M.; Bercovier, H.; Mollaret, H.H. Yersinia enterocolitica among small wild mammals in France. Comp. Immunol. Microbiol. Infect. Dis. 1979, 1, 321-333. [CrossRef]

36. Bancerz-Kisiel, A.; Pieczywek, M.; Łada, P.; Szweda, W. The most important virulence markers of Yersinia enterocolitica and their role during infection. Genes 2018, 9, 235. [CrossRef] 
37. Platt-Samoraj, A.; Syczyło, K.; Szczerba-Turek, A.; Bancerz-Kisiel, A.; Procajło, Z.; Łabuć, S.; Szweda, W. Presence of ail and ystB genes in Yersinia enterocolitica biotype 1A isolates from game animals in Poland. Vet. J. 2017, 221, 11-13. [CrossRef]

38. Joutsen, S.; Johansson, P.; Laukkanen-Ninios, R.; Björkroth, J.; Fredriksson-Ahomaa, M. Two copies of the ail gene found in Yersinia enterocolitica and Yersinia kristensenii. Vet. Microbiol. 2020, 247, 108798. [CrossRef] [PubMed]

39. Joutsen, S.; Laukkanen-Ninios, R.; Henttonen, H.; Niemimaa, H.; Voutilainen, L.; Kallio, L.; Helle, H.; Korkeala, H.; FredrikssonAhomaa, M. Yersinia spp. in wild rodents and shrews in Finland. Vector-Borne Zoonotic Dis. 2017, 17, 303-311. [CrossRef] [PubMed]

40. Fukushima, H.; Gomyoda, M.; Kaneko, S. Mice and moles inhabiting mountainous areas of Shimane Peninsula as sources of infection with Yersinia pseudotuberculosis. J. Clin. Microbiol. 1990, 28, 2448-2455. [CrossRef] [PubMed] 